\title{
Relationship between Concentration Difference of Different Density Lipoproteins and Shear Stress in Atherosclerosis
}

\author{
Wei Meng, ${ }^{1}$ Fengxu Yu, ${ }^{1}$ Huaiqing Chen, ${ }^{2}$ Jianmin Zhang, ${ }^{3}$ Eryong Zhang,, \\ Yingqiang Guo, ${ }^{1}$ and Yingkang Shi ${ }^{1}$ \\ ${ }^{1}$ Department of Thoracic and Cardiovascular Surgery, West China Hospital, Sichuan University, Chengdu 610041, China \\ ${ }^{2}$ Institute of Biomedical Engineering, West China Medical Center, Sichuan University, Chengdu 610041, China \\ ${ }^{3}$ State Key Laboratory of Hydraulics and Mountain River Engineering, Sichuan University, Chengdu 610065, China
}

Correspondence should be addressed to Yingkang Shi, yingkangshi@yahoo.com

Received 15 August 2011; Revised 16 October 2011; Accepted 8 November 2011

Academic Editor: Kyehan Rhee

Copyright (C) 2012 Wei Meng et al. This is an open access article distributed under the Creative Commons Attribution License, which permits unrestricted use, distribution, and reproduction in any medium, provided the original work is properly cited.

\begin{abstract}
Previous research has observed concentration polarization in LDL and HDL in the arterial system. However, there is no report that links this concentration polarization to the development of vascular atherosclerosis (AS). Therefore, the purpose of this study is to establish the relationship between concentration difference of LDL and HDL and shear stress using a carotid bifurcation vascular model. PTFE was employed to create the carotid bifurcation model. Endothelial cells were coated on the inner wall of the graft. In a recirculation system, HDL and LDL concentration were measured under two different ICA flow velocities at 5 different locations within our model. We report the following: (1) LDL and HDL concentration difference was observed in both high flow and low flow environments; (2) the degree of LDL and HDL concentration polarization varied depending of high flow and low flow environment; (3) absolute values of concentration difference between LDL and HDL at the inner wall surface decreased with the increase in shear stress when shear stress was more than $1.5 \mathrm{~Pa}$. This variation trend would be more pronounced if shear stress were less than $0.5 \mathrm{~Pa}$. Our study suggests that under the action of shear stress, concentration differences of LDL or HDL create a disturbance in the balance of atherogenic factors and anti-As factors, resulting in the occurrence of AS.
\end{abstract}

\section{Introduction}

Epidemiology has demonstrated that concentration increment of low-density lipoprotein (LDL) and concentration decrement of high-density lipoprotein (HDL) in blood are independent risk factors for the development of atherosclerosis (AS) $[1,2]$. They assume opposite roles: the former is a harmful lipoprotein while the latter is protective [1-4]. In the majority of cases, AS is found to be located at the bifurcations, bends, or stenosis sites of the artery. In these areas, blood flow is disturbed resulting in flow separation and vortex zone, which is termed localization of AS $[5,6]$. Studies have revealed that reasons resulting in localization of AS include the following: (1) shear stress appearing at the surface of vascular wall and (2) areas of disturbed blood flow leads to its slowing and thus, gives LDL considerable time to interact with the inner wall. As a result, an increasing number of LDL can penetrate the vascular wall.
At present, research has observed concentration polarization in LDL and HDL in the arterial system; these studies also revealed that polarization degree is related to shear stress. Lipid concentration polarization plays an important role in AS development [7-10]. In our research on HDL concentration polarization, we found that under high shear stress circumstance, HDL concentration on the inner surface of vascular wall is inversely proportional to shear stress [10]. This result appeared to contradict with the accepted protective effect of HDL. Based on this observation, the following have been assumed: AS is a result of concerted action of multiple factors and a conclusion cannot be drawn from HDL concentration of the inner surface of vascular wall alone. Under high shear stress circumstance, HDL concentration absolute value will decrease; the protective effect for preventing AS development appears to decrease. However, when integrated consideration with LDL concentration is made, 
can it show the interactive function of concentration polarization of different density lipoproteins in AS development?

On the above-mentioned aspects, we studied the relationship between concentration difference of LDL and HDL on the inner surface of vascular wall and shear stress based on a carotid bifurcation vascular model which designed to mimic the configuration of the human carotid artery [10].

\section{Material and Methods}

2.1. Experimental Model. Semipermeable poly tetra fluoro ethylene (PTFE) was employed to create the carotid bifurcation model. The inner diameter of each region of a human carotid artery was measured by Doppler ultrasound and computerized tomography angiography (CTA) (Figures 2(c) and $2(\mathrm{~d}))$. The measurements were amplified at a rate of $1: 1.5$ (Table 1 ) to create the bifurcation model of carotid artery that was subsequently coated with endothelial cells on the inner wall of the model to induce the endothelialization of the inner wall of the model as shown in Figure $1[10,11]$.

As shown in Figure 2(a), the model included the common carotid artery (CCA), external carotid artery (ECA), internal carotid artery (ICA), and internal carotid artery sinus (ICAS). The low shear stress core region and its margins were marked on the model. The low shear stress core region was marked as point 5 , and the proximal and distal margins were marked as points 3 and 4 (Figure 2(b)). The measuring points of the inner diameter of the CCA and the inner diameter of the ICA were used as control points and marked as points 1 and 2 (Figure 2(b)). In addition, the final model is provided in the schematic diagram in Figure 3(a), and the schematic diagram of the experimental system was shown in Figure 3(b). The whole recirculate system was composed of the upstream reservoir, the model, the flow meter, a centrifugal pump, and the downstream reservoir, connected with tubes. The system is connected with tubing form a complete circuit. A centrifugal pump would pump the fluid from the downstream back to the upper reservoir to establish the circuit. (The fluid was conserved by a reservoir with adjustable height. Before entering the model, the fluid would pass through a segment of straight and horizontal tube. After leaving the model, the fluid flowed forwards to a downstream reservoir through tubes with flow meter. A pump would suck the fluid of the downstream reservoir back to the upper one in order to establish a circulation.) A threshold bar in the upper reservoir could automatically introduce extra fluid back into the downstream container to maintain a stable experimental water stage. The flow direction was shown by arrows (Figure 3(b)).

2.2. Numerical Simulation Methodology. To simplify the analysis, the following assumptions were made: (1) the circulating liquid is incompressible Newtonian fluid; (2) the flow is a steady flow; (3) the wall of bifurcation model is permeable to plasma and has a filtration rate of the order of $10^{6} \mathrm{~cm} / \mathrm{s}$. If the volume power, heat exchange, and other physical and chemical factors are not considered then the equations provided in the footnote can be employed.
TABLE 1: The inner diameter of carotid artery measured by Doppler ultrasound and CTA and the model measurements obtained by amplifying the actual values by 1.5 .

\begin{tabular}{lcc}
\hline Measuring position & $\begin{array}{c}\text { Measuring size } \\
(\mathrm{mm})\end{array}$ & Model size $(\mathrm{mm})$ \\
\hline $\begin{array}{l}\text { Common carotid } \\
\text { Artery (CCA) }\end{array}$ & 6.5 & 9.8 \\
$\begin{array}{l}\text { Internal carotid } \\
\text { Artery (ICA) }\end{array}$ & 4.9 & 7.4 \\
$\begin{array}{l}\text { External Carotid } \\
\text { Artery (ECA) }\end{array}$ & 4.2 & 6.3 \\
$\begin{array}{l}\text { Internal carotid } \\
\text { Artery sinus (ICAS) }\end{array}$ & 7.8 & 11.7 \\
\hline
\end{tabular}

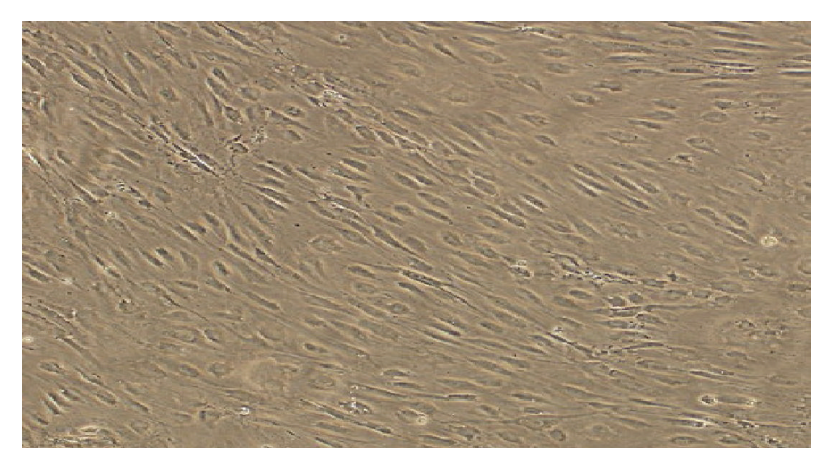

Figure 1: Observation of cell growth and cell arrangement with a converted microscope after implanting the inner surface of the carotid bifurcation model (magnification $\times 100$ ).

The first formula is a continuity equation and the second formula is an equation of motion where $u_{i}$ is the velocity of flow field, $P$ is the fluid pressure, $\rho$ is the fluid density and $\mu$ is the fluid viscosity. Because the basic equations mentioned above are intensive non-linear equations, the Finite Volume Method (FVM) was employed as it is the most commonly used numerical method for resolving this type of mathematical problem at present [12]. The FLUENT software is hydrodynamic calculating software based on the FVM and it is the CFD software applied most widely utilized.

$$
\begin{gathered}
\frac{\partial u_{j}}{\partial x_{j}}=0 \\
\rho \frac{\partial u_{j} u_{i}}{\partial x_{j}}=-\frac{\partial P}{\partial x_{i}}+\mu \frac{\partial}{\partial x_{j}}\left(\frac{\partial u_{i}}{\partial x_{j}}+\frac{\partial u_{j}}{\partial x_{i}}\right) .
\end{gathered}
$$

The boundary conditions are

$$
\begin{aligned}
& u(x, 1, t)=0, \quad v(x, 1, t)=\frac{\mathrm{Vw}}{\overline{\mathrm{U}}_{0}}, \\
& u(0, y, t)=\mathrm{Uwm}, \quad v(0, y, t)=0, \quad c(0, y, t)=1 .
\end{aligned}
$$

The bifurcation model was considered as a semipermeable membrane with a filtration rate of $\mathrm{Vw}$. 


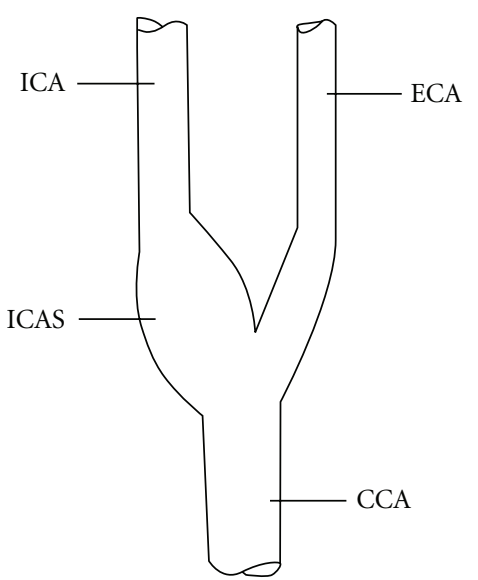

(a)

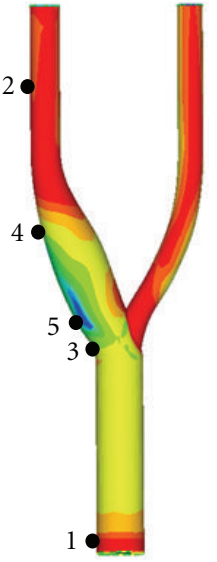

(b)

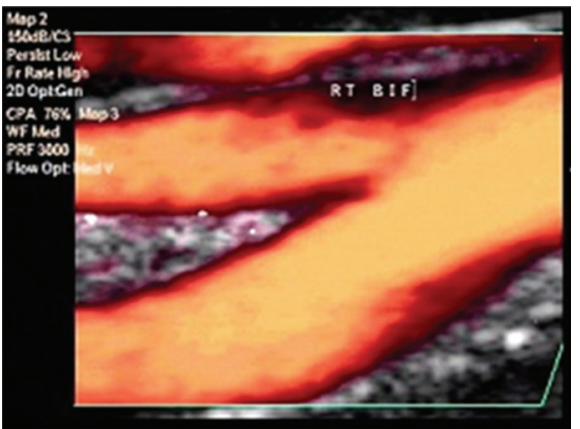

(c)

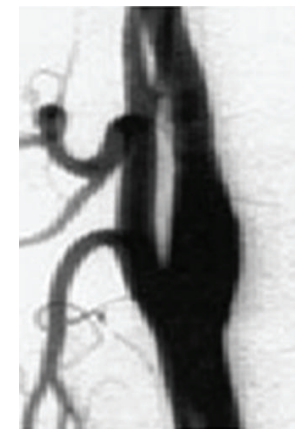

(d)

Figure 2: (a) Pictorial illustration of the carotid bifurcation vascular model. CCA: common carotid artery, ICA: internal carotid artery, ECA: external carotid artery, ICAS: internal carotid artery sinus. (b) The distribution of the shear stress in the carotid bifurcation vascular model. Color changes show the degree of shear stress. Blue represents the lowest shear stress while red is the highest. 1 and 2: control locations of CCA and ICA; 3 and 4: anterior and posterior edges of the low shear stress region; 5: core region of the low shear stress area. (c) The Doppler ultrasound image of carotid artery in long axis plane. (d) The CTA image of carotid artery in coronal plane.
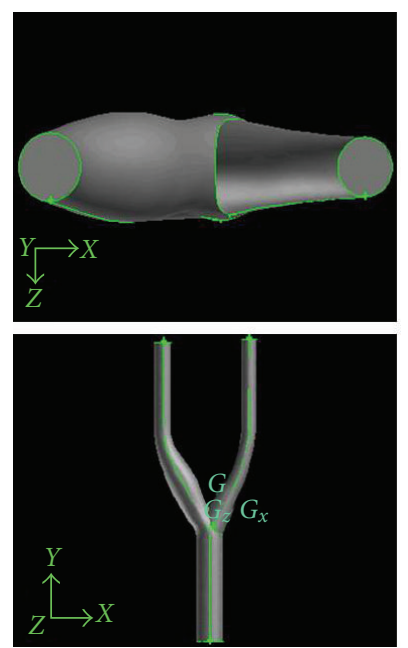

(a)
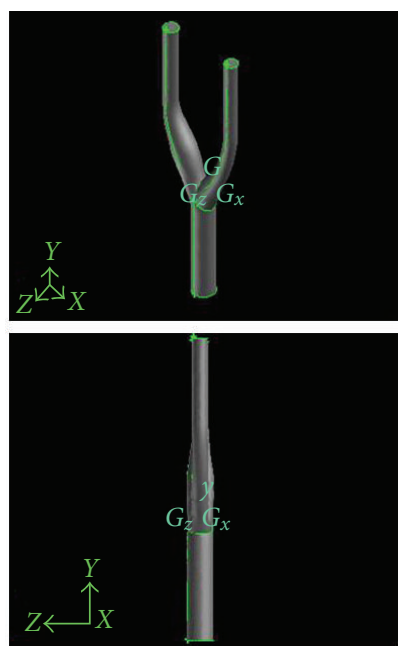

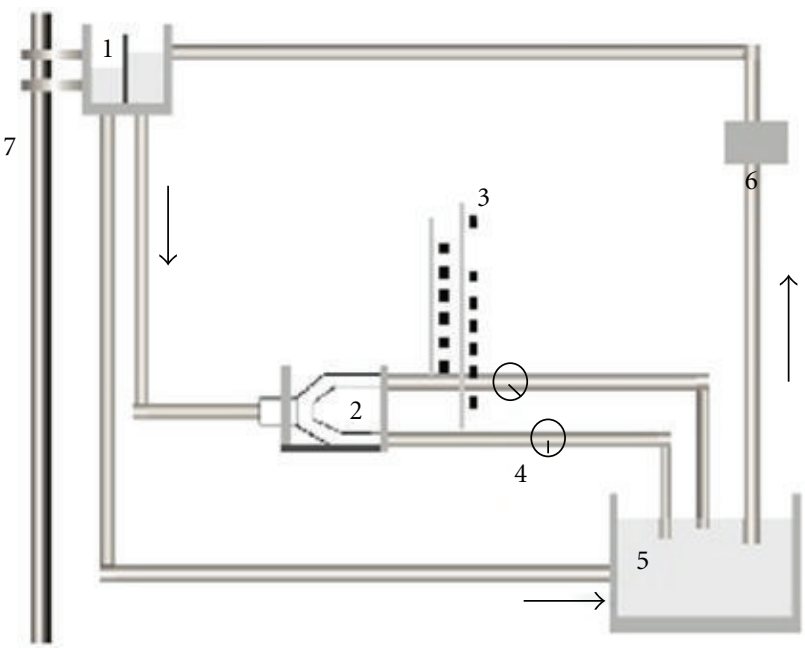

(b)

FIGURE 3: (a) Schematic diagram of the numerical simulation model (four different views angles). (b) The schematic diagram of the experiment recirculation system. Indicating points are (1) upstream reservoir, (2) carotid bifurcation vascular model, (3) manometer, (4) flow meter, (5) downstream reservoir, (6) centrifugal pump, and (7) slide pole.

2.3. Hydrodynamic Parameters of Blood Flow. The parameters of the blood flow in the model were controlled by the particle image velocimetry (PIV) using a type PIV-40010 (TSI Company, Shoreview, MN, USA) and numerical simulation (NS) [13]. The average flow velocity of ICA was set at $0.559 \mathrm{~m} / \mathrm{s}$, which is the average flow velocity within the ICA measured in the human body at $150 \mathrm{mmHg}$ blood pressure.

A solution prepared with $7.5 \%$ glycerol with a viscosity of $0.782 \mathrm{mPa}$.s and a density of $1.005 \times 10^{3} \mathrm{~kg} / \mathrm{m}^{3}$ (for pre run and stabilize the equipment) was measured by the Low Shear 30 (CONTRAVES LOW SHEAR 30 ISCOMETER, Swiss). This viscosity was chosen because it was the same viscosity as the M199 culture medium used for the endothelial cells. Blood flow parameters determined were flow rate $(\mathrm{mL} / \mathrm{s})$ and velocity of the circulation liquid through the model (flow velocity, $\mathrm{m} / \mathrm{s}$ ).

2.4. Separation of HDL and LDL. Human plasma lipoproteins were collected and separated using the one-time density gradient ultracentrifugation method described by Zhang and Liu [14]. HDL and LDL bands were collected from centrifuged samples using a long syringe needle and were dialyzed in a buffer containing $0.02 \mathrm{~mol} / \mathrm{L}$ Tris- $\mathrm{HCl}, 0.85 \%$ $\mathrm{NaCl}, 0.01 \%$ EDTA, and $0.01 \% \mathrm{NaN}_{3}$ at $\mathrm{pH}$ 7.6. Dialysis was performed at $4^{\circ} \mathrm{C}$ in the dark for 6 hours each time 


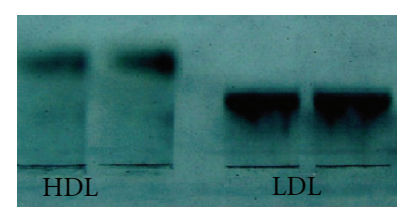

Figure 4: Purity of HDL and LDL banding patterns in gel electrophoresis after density gradient ultracentrifugation.

and completely repeated 4 times in order to remove sodium bromide. Collected lipoproteins were stored at $4^{\circ} \mathrm{C}$ following filtration (storage and dialysis were performed under a nitrogen atmosphere to avoid oxidation). Figure 4 shows the high purity of the isolated LDLs and HDLs. In total, $100 \mathrm{~mL}$ of circulation liquid was prepared for the experiments, including $80 \mathrm{ml} \mathrm{M} 199$ medium and $20 \mathrm{~mL}$ of separated human plasma lipoproteins (i.e., $10 \mathrm{~mL}$ LDL and $10 \mathrm{~mL}$ HDL). Lipoprotein concentrations were determined with an OLYMPUS automatic biochemical analyzer (OLYMPUS automatic biochemical analyzer AU2700, Japan). The concentrations of LDL and HDL in the bulk flow $\left(C_{0}\right)$ were $0.575 \mathrm{mmol} / \mathrm{L}$ and $0.242 \mathrm{mmol} / \mathrm{L}$, respectively.

2.5. Experimental Procedure. HDL and LDL concentration were measured under two different ICA flow velocities at 5 different locations in the model. The low-speed group had an average ICA flow velocity of $0.559 \mathrm{~m} / \mathrm{s}$ (the average flow velocity of ICA in human body under $150 \mathrm{mmHg}$ blood pressure), while the high-speed group had an average ICA flow velocity of $1.451 \mathrm{~m} / \mathrm{s}$ (the peak flow velocity of ICA in human body under $90 \mathrm{mmHg}$ blood pressure). Hydrodynamic parameters (i.e., blood flow in the low- and high-speed groups) were measured and have been summarized in Table 2.

After allowing the model to stabilize for 30 minutes, $50 \mu \mathrm{L}$ samples were sequentially collected from each of the 5 locations and 5 samples were collected consecutively from each location. The samples were collected 15 minutes apart to ensure that samples were collected at a constant flow. The collected samples were individually placed in polyethylene tubes and stored protected from light in brown bottles at $4^{\circ} \mathrm{C}$. Lipoprotein concentrations were measured within 4 hours of collection. The ratio of the concentration of LDL at the surface $\left(C_{\mathrm{IS}}\right)$ to the concentration in bulk $\left(C_{10}\right)$ was used as an index for concentration polarization of LDL. The ratio of the concentration of HDL at the surface $\left(C_{\mathrm{hS}}\right)$ to the concentration in bulk $\left(C_{\mathrm{h} 0}\right)$ was used as an index for concentration polarization of HDL. Polarization of LDL and HDL was considered to have occurred if the ratio was greater than 1.000 .

2.6. Statistical Analysis. The experimental result is shown in mean number \pm standard deviation $(\bar{x} \pm s)$. Comparison between multiple groups was performed using one-factor analysis of variance, whereas comparison between two groups involved the $t$-test. Data correlation contrast analysis was performed using linear regression. The result was deemed as a significant difference when $P$ was below 0.05 .
TABLE 2: Hydrodynamic parameters for low-speed and high-speed flow groups.

\begin{tabular}{lcc}
\hline Group & Flow rate $(\mathrm{mL} / \mathrm{s})$ & Flow velocity $(\mathrm{m} / \mathrm{s})$ \\
\hline Low-Speed & 60.62 & 0.559 \\
High-speed & 155.93 & 1.451 \\
\hline
\end{tabular}

All statistical analyses were computed using the SPSS11.5 statistics package.

\section{Results}

\subsection{The Surface Concentration of LDL and HDL at Low-Speed} Flow Group. See (Table 3).

3.2. The Surface Concentration of LDL and HDL at HighSpeed Flow Group. From Tables 3 and 4, LDL and HDL concentration polarization on the inner surface of carotid artery bifurcation model was observed at every sampling point in both two groups.

3.3. Relationship between Concentration Difference of HDL and LDL and Shear Stress. LDL and HDL concentration difference on the inner surface of carotid artery bifurcation model was observed.

Absolute values of concentration difference between LDL and HDL on each sampling point of low- and high-speed flow groups (Table 5) were compared. No statistic difference was observed when absolute values of concentration difference between LDL and HDL on Sampling Points 1 and 2 were compared. However, statistical difference was noted by comparing absolute values of concentration difference between LDL and HDL on Sampling Points 3, 4, and 5; P value was less than 0.05 .

Comparing the ratio of LDL concentration at the inner wall surface on each sampling point of low- and high-speed flow group to concentration in bulk flow and the ratio of HDL concentration at the inner wall surface to concentration in bulk flow, the difference value showed the following: ratio of LDL concentration at the inner wall surface in low-speed flow to concentration in bulk flow was significantly higher than HDL; the difference between these two values was a positive value. However, the ratio of LDL concentration at the inner wall surface in high-speed flow to concentration in bulk flow was significantly lower than HDL; the difference between these two values was a negative value. Statistical difference was observed by comparing difference between the ratios of LDL and HDL concentration at the inner wall surface from these two groups to concentration in bulk flow.

As demonstrated in Figure 5, absolute values of concentration difference between LDL and HDL at the inner wall surface decreased with the increase in shear stress when shear stress was less than $1.5 \mathrm{~Pa}$. This variation trend would be more pronounced if shear stress were less than $0.5 \mathrm{~Pa}$. After shear stress increased to $1.5 \mathrm{~Pa}$, absolute values of concentration difference between LDL and HDL at the inner surface of wall remained unchanged according to shear stress. 
TABLE 3: The surface concentration of LDL and HDL at low-speed flow group.

\begin{tabular}{|c|c|c|c|c|c|}
\hline Sampling point & Shear stress $(\mathrm{Pa})$ & $C_{\mathrm{ls}}(\mathrm{mmol} / \mathrm{L})(n=5)$ & $C_{\mathrm{ls}} / C_{l 0}$ & $C_{\mathrm{hs}}(\mathrm{mmol} / \mathrm{L})(n=5)$ & $C_{\mathrm{hs}} / C_{\mathrm{h} 0}$ \\
\hline 1 & 1.921 & $0.576 \pm 0.015$ & 1.011 & $0.242 \pm 0.005$ & 1.008 \\
\hline 2 & 1.537 & $0.581 \pm 0.033$ & 1.049 & $0.246 \pm 0.006$ & 1.025 \\
\hline 3 & 0.448 & $0.696 \pm 0.013^{\star \star \#}$ & $1.221^{\star \star \#}$ & $0.276 \pm 0.005^{\star \#}$ & $1.150^{\star \star \#}$ \\
\hline 4 & 0.297 & $0.744 \pm 0.018^{\star \star \Delta}$ & $1.305^{\star \star \Delta}$ & $0.304 \pm 0.008^{\star \star \Delta}$ & $1.292^{\star \star \Delta}$ \\
\hline 5 & 0.069 & 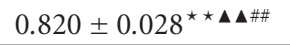 & 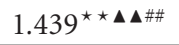 & 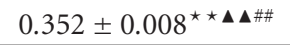 & $1.407^{\star \star \Delta \Delta \# \#}$ \\
\hline
\end{tabular}

${ }^{\star} P<0.05$ versus points 1 and $2,{ }^{\star \star} P<0.01$ versus points 1 and 2 .

$\mathbf{\Delta} P<0.05$ versus point $3, \mathbf{\Delta} \mathrm{\Delta} P 0.01$ versus point 3

${ }^{\#} P<0.05$ versus point 4 , ${ }^{\#} P<0.01$ versus point 4 .

$C_{\mathrm{ls}}:$ LDL concentration at surface.

$C_{\mathrm{hs}}$ : HDL concentration at surface.

$C_{10}$ : LDL concentration in bulk flow.

$C_{\mathrm{h} 0}$ : HDL concentration in bulk flow.

TABLE 4: The surface concentration of LDL and HDL at high-speed flow group.

\begin{tabular}{lccccc}
\hline Sampling point & Shear stress $(\mathrm{Pa})$ & $C_{\mathrm{ls}}(\mathrm{mmol} / \mathrm{L})(n=5)$ & $C_{\mathrm{ls}} / C_{\mathrm{l} 0}$ & $C_{\mathrm{hs}}(\mathrm{mmol} / \mathrm{L})(n=5)$ & $C_{\mathrm{hs}} / C_{\mathrm{h} 0}$ \\
\hline 1 & 5.833 & $0.576 \pm 0.015$ & 1.011 & $0.242 \pm 0.004$ & 1.008 \\
2 & 4.661 & $0.584 \pm 0.006$ & 1.025 & $0.244 \pm 0.006$ & 1.017 \\
3 & 2.329 & $0.584 \pm 0.152$ & 1.025 & $0.250 \pm 0.012$ & $1.041^{*}$ \\
4 & 1.334 & $0.592 \pm 0.130 \Delta$ & $1.042 \Delta$ & $0.252 \pm 0.008$ & $1.050^{*}$ \\
5 & 0.896 & $0.614 \pm 0.151^{*} \Delta \star \star \Delta \mathbf{\Delta} *$ & $1.077 \Delta \Delta \star \Delta \#$ & $0.262 \pm 0.008^{* * \Delta}$ & $1.092^{* * \Delta \Delta *}$ \\
\hline
\end{tabular}

$\triangle P<0.05$ versus point $1, \Delta \triangle P<0.01$ versus point 1 .

${ }^{\star} P<0.01$ versus points $2,{ }^{\star \star} P<0.01$ versus points 2 .

${ }^{*} P<0.01$ versus points 1 and $2,{ }^{* *} P<0.01$ versus points 1 and 2 .

$\Delta P<0.05$ versus point $3, \boldsymbol{\Delta} \Delta P<0.01$ versus point 3 .

${ }^{\#} P<0.05$ versus point $4,{ }^{\# \#} P<0.01$ versus point 4 .

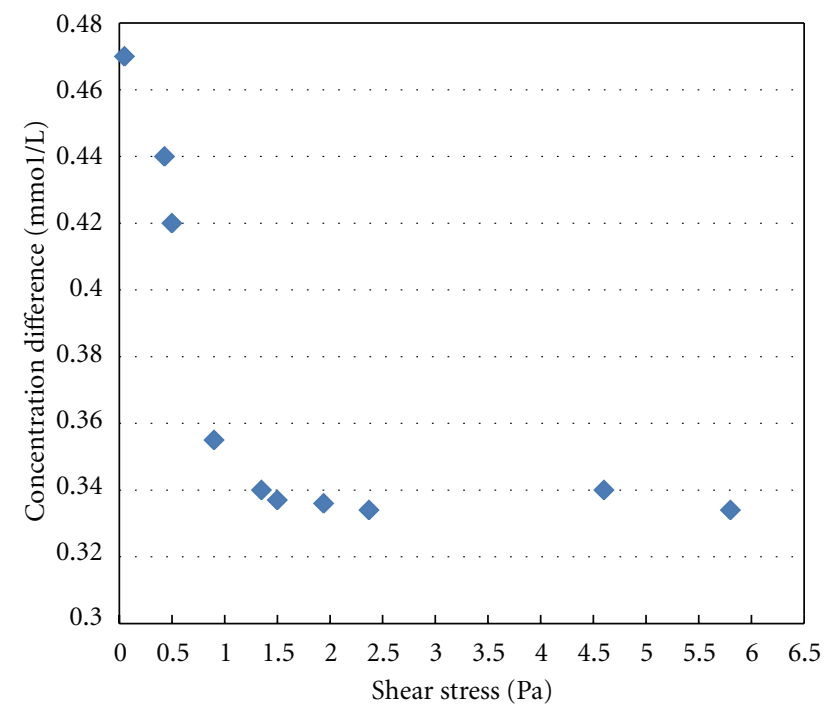

FIGURE 5: Relationship between concentration difference of HDL and LDL and shear stress.

Statistics revealed that on the inner wall surface of ICAS, absolute values of concentration difference between LDL and HDL at the inner wall surface were negatively correlated with shear stress $(r=-29.386, P=0.022)$. After shear stress increased to $1.5 \mathrm{~Pa}$, concentration difference between
LDL and HDL at the inner wall surface was maintained at a relatively constant lower level. No negative correlation was observed.

Results revealed a concentration difference between LDL and HDL at the inner wall surface, particularly on the inner wall surface of ICAS.

\section{Discussion}

Concentration increment of LDL and concentration decrement of HDL are independent risk factors for the development of AS. They assume opposite roles. While the former is a harmful lipoprotein, the latter is a protective one. The major reasons for the anti-AS mechanism of HDL are as follows: participating reverse cholesterol transcription, antiLDL oxidation, and the protection of endothelial cells [1522]. In the atherogenic mechanism of LDL, there are more $\operatorname{ROS}\left(\mathrm{O}_{2}^{-}, \mathrm{H}_{2} \mathrm{O}_{2}\right.$ and ect) in the area with low shear stress because of increased expression of NADPH oxidase and defect in oxygen transfer. This accelerates LDL oxidation and generates Ox-LDL. Ox-LDL can induce expression of vascular endothelial cell MCP-1 to result in adhesion and migration of monocyte. Similarly, it can reduce expressions of antiapoptotic proteins, Bcl-2, and c-IAP-1, by combining specific receptors LOX-1 to induce cell apoptosis. Thus, concentration polarization of LDL provides pathogenic OxLDL with sufficient substrate, and in the setting of low shear stress environment, makes the endothelial cells in these 
TABLE 5: Concentration difference between LDL and HDL on each sampling point on the inner vascular wall surface of carotid artery bifurcation model.

\begin{tabular}{lcccc}
\hline Sampling points & $\begin{array}{c}\mathrm{CH}_{\mathrm{LDL}}-\mathrm{CH}_{\mathrm{HDL}}(\mathrm{mmol} / \mathrm{L}) \\
(n=5)\end{array}$ & $\begin{array}{c}\mathrm{RH}_{\mathrm{LDL}}-\mathrm{RH}_{\mathrm{HDL}} \\
(n=5)\end{array}$ & $\begin{array}{c}\mathrm{Cl}_{\mathrm{LDL}}-\mathrm{Cl}_{\mathrm{HDL}} \\
(\mathrm{mmol} / \mathrm{L})(n=5)\end{array}$ & $\begin{array}{c}\mathrm{RL}_{\mathrm{LDL}}-\mathrm{RL}_{\mathrm{HDL}} \\
(n=5)\end{array}$ \\
\hline 1 & $0.334 \pm 0.012$ & 0.003 & $0.334 \pm 0.029$ & 0.003 \\
2 & $0.340 \pm 0.016$ & 0.008 & $0.335 \pm 0.017$ & 0.024 \\
3 & $0.334 \pm 0.015$ & -0.016 & $0.420 \pm 0.017^{\star \star}$ & $0.071^{\star}$ \\
4 & $0.340 \pm 0.016$ & -0.008 & $0.440 \pm 0.015^{\star \star}$ & $0.013^{\mathbf{\Delta}}$ \\
5 & $0.352 \pm 0.020$ & -0.015 & $0.468 \pm 0.030^{\star \star}$ & $0.032^{\mathbf{\Delta}}$ \\
\hline
\end{tabular}

$\mathrm{CH}_{\mathrm{LDL}}-\mathrm{CH}_{\mathrm{HDL}}$ : Concentration difference between LDL and HDL at the inner wall surface in high-speed flow group.

$\mathrm{CL}_{\mathrm{LDL}}-\mathrm{CL}_{\mathrm{HDL}}$ : Concentration difference between LDL and HDL at the inner wall surface in low-speed flow group.

$\mathrm{RH}_{\mathrm{LDL}}-\mathrm{RH} \mathrm{HDL}_{\mathrm{L}}$ : Difference value of ratio of concentration between LDL and HDL at the inner wall surface in high-speed flow to concentration in bulk flow. $\mathrm{RL}_{\mathrm{LDL}}-\mathrm{RL} \mathrm{HDL}$ : Difference value of ratio of concentration between LDL and HDL at the inner wall surface in low-speed flow to concentration in bulk flow.

$\boldsymbol{\Delta} P<0.05$ versus $\mathrm{RH}_{\mathrm{LDL}}-\mathrm{RH}_{\mathrm{HDL}},{ }^{\boldsymbol{\Delta}} \mathrm{\Delta} P<0.01$ versus $\mathrm{RH}_{\mathrm{LDL}}-\mathrm{RH}_{\mathrm{HDL}} ;{ }^{\star \star} P<0.01$ versus $\mathrm{CH}_{\mathrm{LDL}}-\mathrm{CH}_{\mathrm{HDL}}$.

areas more vulnerable to the damage, and thus facilitates occurrence of atherosclerosis [23-26].

Concentration polarization degree of HDL and LDL is correlated with shear stress. However, the degree of HDL polarization and LDL polarization is different under different shear stress. As evidenced in Table 5, under high-speed flow circumstance, shear stress at the inner wall surface of ICAS (sampling point 5) increased when low-speed flow was compared, and the LDL concentration polarization degree was lower than HDL. This indicated that concentration polarization degree of lipoprotein with different molecular weight and size is different under different shear stress. We have furthered the study on this phenomenon and proposed the concept of "concentration difference" between HDL and LDL.

According to the phenomenon observed in experiments, we compared the absolute values of LDL-HDL concentration difference at the wall surfaces of every point in low- and highspeed flow with difference value of ratio of concentration in bulk flow. Statistical results revealed that in CCA and ICA (sampling points 1 and 2), no obvious difference in absolute values of concentration difference was observed. However, in ICAS, absolute value of concentration difference between LDL and HDL at the inner wall surface in low-speed flow was significantly higher than that in high-speed flow; in the ICAS area with wall shear stress less than $0.5 \mathrm{~Pa}$, the absolute value of concentration difference between LDL and HDL at the inner wall surface was negatively correlated with shear stress. In ICAS, a significant difference was observed between the difference value of ratios of LDL and HDL concentration at the inner wall surface in high- and lowspeed flow to concentration in bulk flow. In low-speed flow, wall shear stress in ICAS part was lower, the difference value between the ratio of concentration of LDL at the inner wall surface to concentration in bulk flow and ratio of concentration of HDL to concentration in bulk flow was a positive value. In high-speed flow, wall shear stress in ICAS was obviously increased, the difference value between the ratio of concentration of LDL at the inner wall surface to concentration in bulk flow and ratio of concentration of HDL to concentration in bulk flow was a negative value. This indicated that concentration polarization degree of LDL was higher than HDL in low-speed flow and low shear stress, and concentration polarization degree of HDL was higher than LDL in high-speed flow and high shear stress.

We first proposed that in the situation with certain low shear stress in the flow field, concentration polarization at the inner wall surface of lipoproteins with different molecular weights would change along with change in shear stress, resulting in inconsistent concentration profile; this is referred to as concentration difference. According to our study on concentration difference, in low stress situations, the increase in harmful LDL outweighs the increase in protective HDL. Other studies have suggested that the occurrence probability of AS angiocardiopathy would increase by $1 \%-2 \%$ if LDL level increased by $1 \%$ or if HDL level reduced by $1 \%[27,28]$.

\section{Conclusion}

In conclusion, we propose that the formation of AS may be secondary to an imbalance between the proatherosclerotic LDL and the antiatherosclerotic HDL. We demonstrated that in low stress environment, the increase in LDL outweighs the increase in HDL leading to AS. On the other hand, in high stress environment, the increases in HDL outweighs the increase in LDL which leads to a protective effect on atherosclerosis.

\section{Authors' Contribution}

Y. Guo and Y. Shi contributed equally to this paper.

\section{Acknowledgment}

This Work Was Supported by the National Natural Science Foundation of China (81170288).

\section{References}

[1] A. M. Gotto Jr. and E. A. Brinton, "Assessing low levels of highdensity lipoprotein cholesterol as a risk factor in coronary heart disease: a working group report and update," Journal of the American College of Cardiology, vol. 43, no. 5, pp. 717-724, 2004. 
[2] A. von Eckardstein and G. Assmann, "Prevention of coronary heart disease by raising high-density lipoprotein cholesterol?" Current Opinion in Lipidology, vol. 11, no. 6, pp. 627-637, 2000.

[3] J. Chen, J. L. Mehta, N. Haider, X. Zhang, J. Narula, and D. $\mathrm{Li}$, "Role of caspases in Ox-LDL-induced apoptotic cascade in human coronary artery endothelial cells," Circulation Research, vol. 94, no. 3, pp. 370-376, 2004.

[4] J. R. Nofer, M. Walter, and G. Assmann, "Current understanding of the role of high-density lipoproteins in atherosclerosis and senescence," Expert Review of Cardiovascular Therapy, vol. 3, no. 6, pp. 1071-1086, 2005.

[5] C. G. Caro, J. M. Fitz-Gerald, and R. C. Schroter, "Arterial wall shear and distribution of early atheroma in man," Nature, vol. 223, no. 5211, pp. 1159-1161, 1969.

[6] C. G. Caro, J. M. Fitz-Gerald, and R. C. Schroter, "Atheroma and arterial wall shear. Observation, correlation and proposal of a shear dependent mass transfer mechanism for atherogenesis," Proceedings of the Royal Society of London B, vol. 177, no. 46, pp. 109-159, 1971.

[7] S. Wada and T. Karino, "Theoretical study on flow-dependent concentration polarization of low density lipoproteins at the luminal surface of a straight artery," Biorheology, vol. 36, no. 3, pp. 207-223, 1999.

[8] S. Wada and T. Karino, "Theoretical prediction of low-density lipoproteins concentration at the luminal surface of an artery with a multiple bend," Annals of Biomedical Engineering, vol. 30, no. 6, pp. 778-791, 2002.

[9] G. Wang, X. Deng, and R. Guidoin, "Concentration polarization of macromolecules in canine carotid arteries and its implication for the localization of atherogenesis," Journal of Biomechanics, vol. 36, no. 1, pp. 45-51, 2003.

[10] W. Meng, F. Yu, H. Chen et al., "Concentration polarization of high-density lipoprotein and its relation with shear stress in an in vitro model," Journal of Biomedicine and Biotechnology, vol. 2009, Article ID 695838, 8 pages, 2009.

[11] P. Zilla, M. Deutsch, J. Meinhart et al., "Clinical in vitro endothelialization of femoropopliteal bypass grafts: an actuarial follow-up over three years," Journal of Vascular Surgery, vol. 19, no. 3, pp. 540-548, 1994.

[12] Y. Mohammed and J. F. Verhey, "A finite element method model to simulate laser interstitial thermotherapy in anatomical inhomogeneous regions," BioMedical Engineering Online, vol. 4, article 2, 2005.

[13] F. Yu, Y. Shi, W. Deng, H. Chen, Q. An, and Y. Guo, "Particle image velocimetry in measuring the flow fields distribution in carotid artery bifurcation model," Sheng Wu Yi Xue Gong Cheng Xue Za Zhi, vol. 24, no. 1, pp. 104-109, 2007 (Chinese).

[14] L. H. Zhang and B.W. Liu, "Isolation of human plasma lipoprotiens by one-time density gradient ultracentrifugation method," Acta Biochimica et Biophysica Sinica, vol. 21, pp. 257 260, 1989.

[15] P. J. Barter and K. A. Rye, "Molecular mechanisms of reverse cholesterol transport," Current Opinion in Lipidology, vol. 7, no. 2, pp. 82-87, 1996.

[16] E. Boisfer, D. Stengel, D. Pastier et al., "Antioxidant properties of HDL in transgenic mice overexpressing human apolipoprotein A-II," The Journal of Lipid Research, vol. 43, no. 5, pp. 732 741, 2002.

[17] P. Dimayuga, J. Zhu, S. Oguchi et al., "Reconstituted HDL containing human apolipoprotein A-1 reduces VCAM-1 expression and neointima formation following periadventitial cuffinduced carotid injury in apoE null mice," Biochemical and
Biophysical Research Communications, vol. 264, no. 2, pp. 465468, 1999.

[18] H. Hattori, T. Kujiraoka, T. Egashira et al., "Association of coronary heart disease with pre- $\beta$-HDL concentrations in Japanese men," Clinical Chemistry, vol. 50, no. 3, pp. 589-595, 2004.

[19] X. P. Li, S. P. Zhao, X. Y. Zhang, L. Liu, M. Gao, and Q. C. Zhou, "Protective effect of high density lipoprotein on endothelium-dependent vasodilatation," International Journal of Cardiology, vol. 73, no. 3, pp. 231-236, 2000.

[20] M. Navab, S. Y. Hama, G. M. Anantharamaiah et al., "Normal high density lipoprotein inhibits three steps in the formation of mildly oxidized low density lipoprotein: steps 2 and 3," The Journal of Lipid Research, vol. 41, no. 9, pp. 1495-1508, 2000.

[21] J. R. Nofer, B. Levkau, I. Wolinska et al., "Suppression of endothelial cell apoptosis by high density lipoproteins (HDL) and HDL-associated lysosphingolipids," The Journal of Biological Chemistry, vol. 276, no. 37, pp. 34480-34485, 2001.

[22] I. S. Yuhanna, Y. Zhu, B. E. Cox et al., "High-density lipoprotein binding to scavenger receptor-BI activates endothelial nitric oxide synthase," Nature Medicine, vol. 7, no. 7, pp. 853857, 2001.

[23] J. Hwang, M. H. Ing, A. Salazar et al., "Pulsatile versus oscillatory shear stress regulates NADPH oxidase subunit expression: implication for native LDL oxidation," Circulation Research, vol. 93, no. 12, pp. 1225-1232, 2003.

[24] D. W. Crawford, L. H. Back, and M. A. Cole, "In vivo oxygen transport in the normal rabbit femoral arterial wall," The Journal of Clinical Investigation, vol. 65, no. 6, pp. 1498-1508, 1980.

[25] J. A. Berliner, M. C. Territo, A. Sevanian et al., "Minimally modified low density lipoprotein stimulates monocyte endothelial interactions," The Journal of Clinical Investigation, vol. 85, no. 4, pp. 1260-1266, 1990.

[26] J. Chen, J. L. Mehta, N. Haider, X. Zhang, J. Narula, and D. $\mathrm{Li}$, "Role of caspases in Ox-LDL-induced apoptotic cascade in human coronary artery endothelial cells," Circulation Research, vol. 94, no. 3, pp. 370-376, 2004.

[27] D. J. Gordon, J. L. Probstfield, R. J. Garrison et al., "Highdensity lipoprotein cholesterol and cardiovascular disease. Four prospective American studies," Circulation, vol. 79, no. 1, pp. 8-15, 1989.

[28] P. W. Wilson, K. M. Anderson, W. P. Castelli, and W. B. Kannel, "Twelve-year incidence of coronary heart disease in middle-aged adults during the era of hypertensive therapy: the Framingham Offspring Study," The American Journal of Medicine, vol. 90, no. 1, pp. 11-16, 1991. 


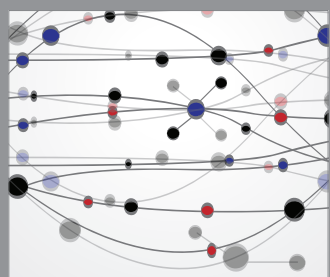

The Scientific World Journal
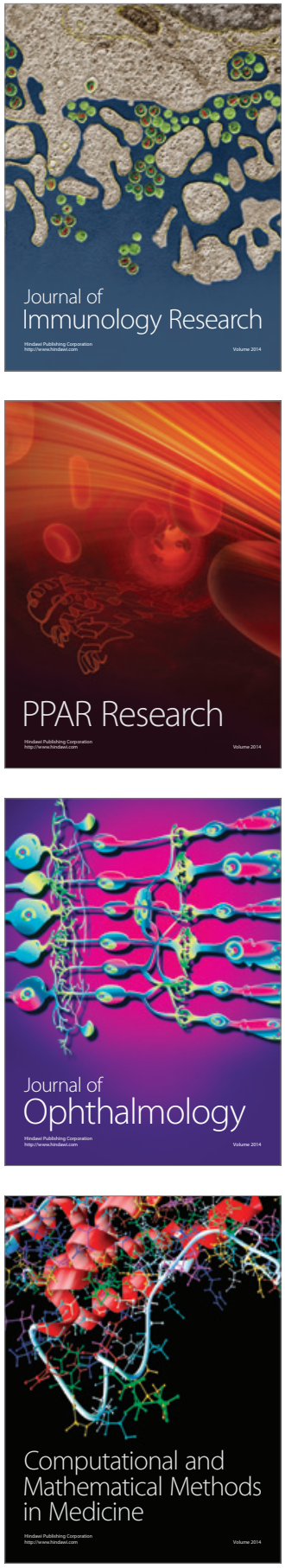

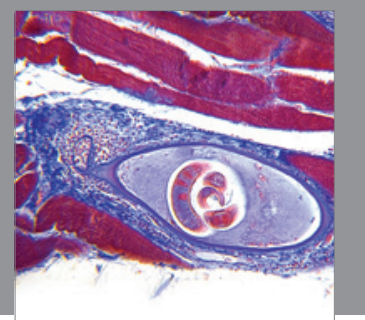

Gastroenterology

Research and Practice
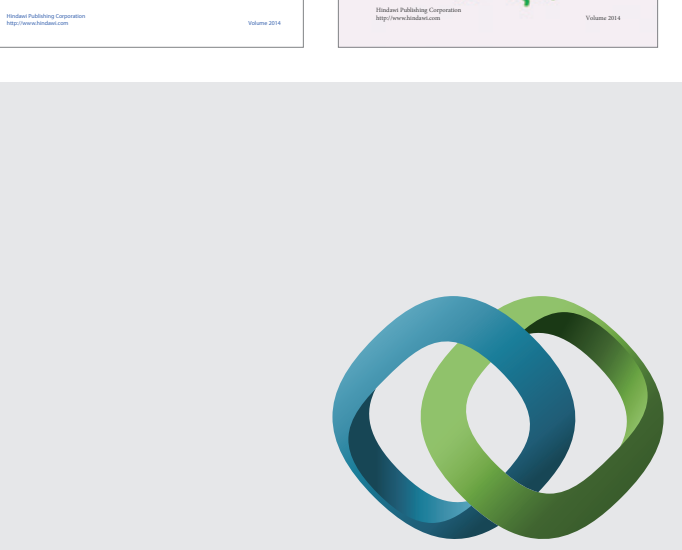

\section{Hindawi}

Submit your manuscripts at

http://www.hindawi.com
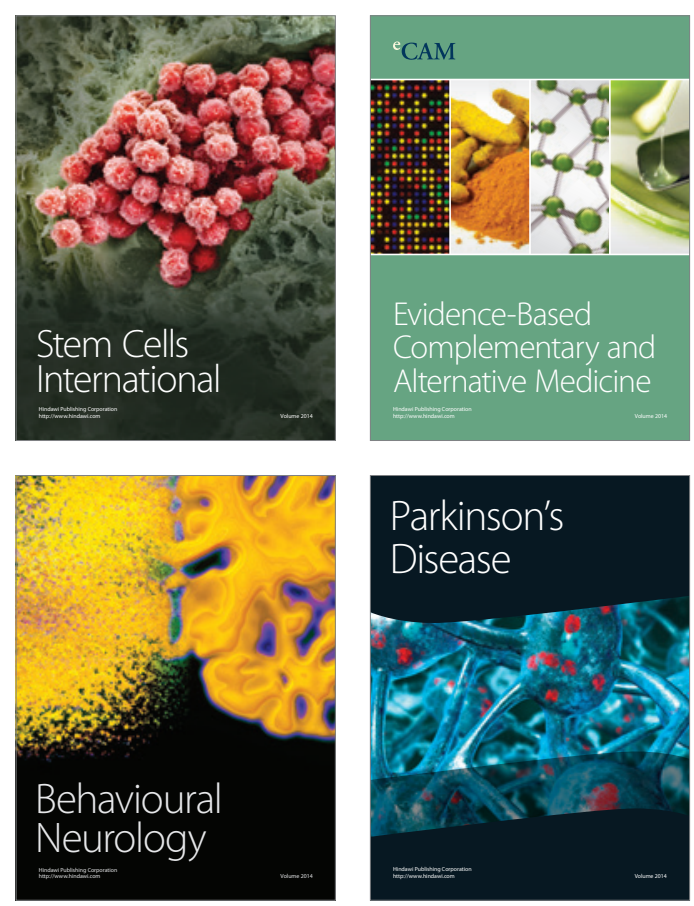

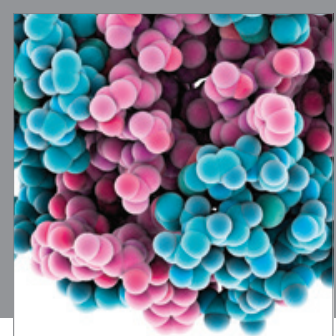

Journal of
Diabetes Research

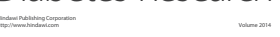

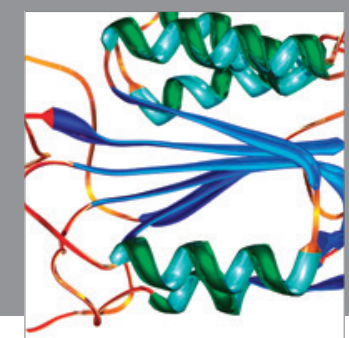

Disease Markers
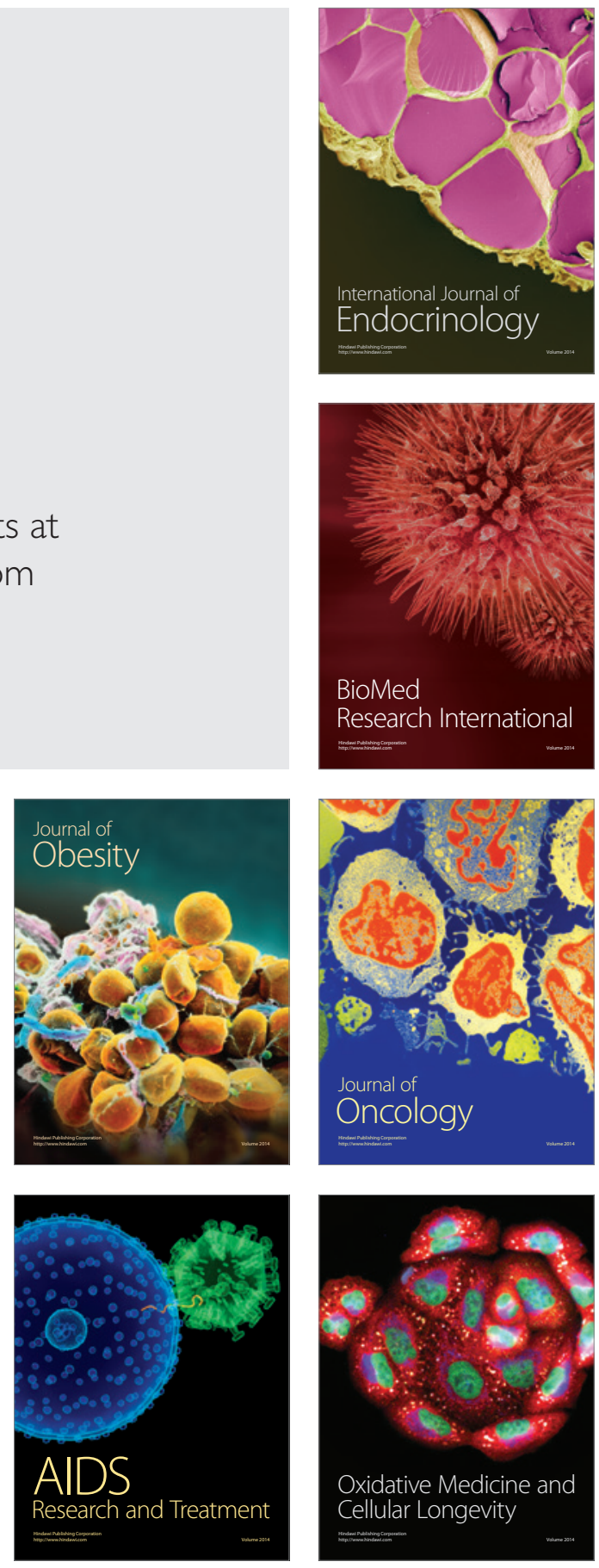